\title{
EVENT-IMPORTANCE BASED CUSTOMIZED AND AUTOMATIC CRICKET HIGHLIGHT GENERATION
}

\author{
Maheshkumar H. Kolekar, Student Member, IEEE and Somnath Sengupta, Member, IEEE \\ Department of Electronics and Electrical Communication Engineering \\ Indian Institute of Technology Kharagpur-721302 (India)
}

\begin{abstract}
In this paper, we present a novel approach towards customized and automated generation of sports highlights from its extracted events and semantic concepts. A recorded sports video is first divided into slots, based on the game progress and for each slot, an importance-based concept and event-selection is proposed to include those in the highlights. Using our approach, we have successfully extracted highlights from recorded video of cricket match.
\end{abstract}

\section{INTRODUCTION}

With the phenomenal growth of digital video contents, there is a need to prune the recorded video contents to extract only the events, which are more important than the rest. This is especially a major requirement for sports video, where the process of pruning and highlight generation have been through manual involvement so far. Manual highlight generation is a tedious process for long-duration games, such as cricket.

In recent years, there have been some efforts towards automated generation of sports highlights. The techniques adopted so far can be broadly classified as event-based [1], [2], or excitement model-based, through the pioneering work of Hanjalic [3]. The event-based video abstraction techniques mostly utilize some of the recent developments on event-detection, which may be based on feature-based event models [4] or inter-event collaboration [5]. Event-based techniques reported in literature so far, suffer from a limitation that events having not-so-obvious keywords, but which may be of interest to the viewers, may be missed out. Excitement-model based highlight generation technique of Hanjalic looks for excitement through features such as motion activity, cut density and sound energy. However, his approach may include some offfield distractions in the highlights, which may not be of interests to the viewers. An automated sports highlight generation technique should have a better link with the sports semantics to avoid such problems. Excitement-based highlight generation has no direct link with the semantics. On the other hand, event-based highlights use more semantic contents than the former and its success depends on the richness of the semantic concepts.
Our work on highlight generation is based on events that are grouped into rich semantic concepts, using our earlier reported works on hierarchical classification and semantic concept extraction framework [6]. In contrast with Hanjalic's, our work has addressed the excitement extraction in the first level of our hierarchical framework.

The main contribution of this paper is the novel technique of selecting semantic concepts and the events within the concepts, according to their degree of importance. Different importance may be assigned by different group of users. For example, a general viewer may like to have a comprehensive viewing of all important actions, whereas, specialist viewers may like to observe actions of their choice. Our approach facilitates such customized highlight generation, by assigning event importances.

In our implementation and experimentation, we considered cricket for highlight generation. Cricket is a popular game in at least ten nations. Its structuring is altogether different from those of tennis, baseball, soccer, football etc, on which several works have been reported. However, cricket has not been properly addressed yet for automatic semantic concept extraction and highlight generation. The reasons are possibly the increased complexity of the game and especially the long durations, for which highly efficient video pruning is required.

\section{AUTOMATED HIGHLIGHT GENERATION SYSTEM}

Our approach uses two level of abstractions- one at the microlevel, called events and the other at the macrolevel, called concepts. Events may be defined as shots with some semantic meaning attached to it. Events are extracted as the leaf nodes of a hierarchical feature-based classifier [6], [7]. In cricket, examples of specific events are real-time field actions, fielders' celebrations, replays etc.

Concepts, more appropriately known as the semantic concepts, are more domain-specific and are derived from the events through a semantic rule-base. Semantic concepts are the collection of a temporally ordered set of events. Semantic concepts relevant to cricket are wicket, hit, special moments (like achieving milestones) etc. 
Each concept $C_{i}$ is a collection of events $E_{1}, E_{2} \ldots \ldots E_{m}$,

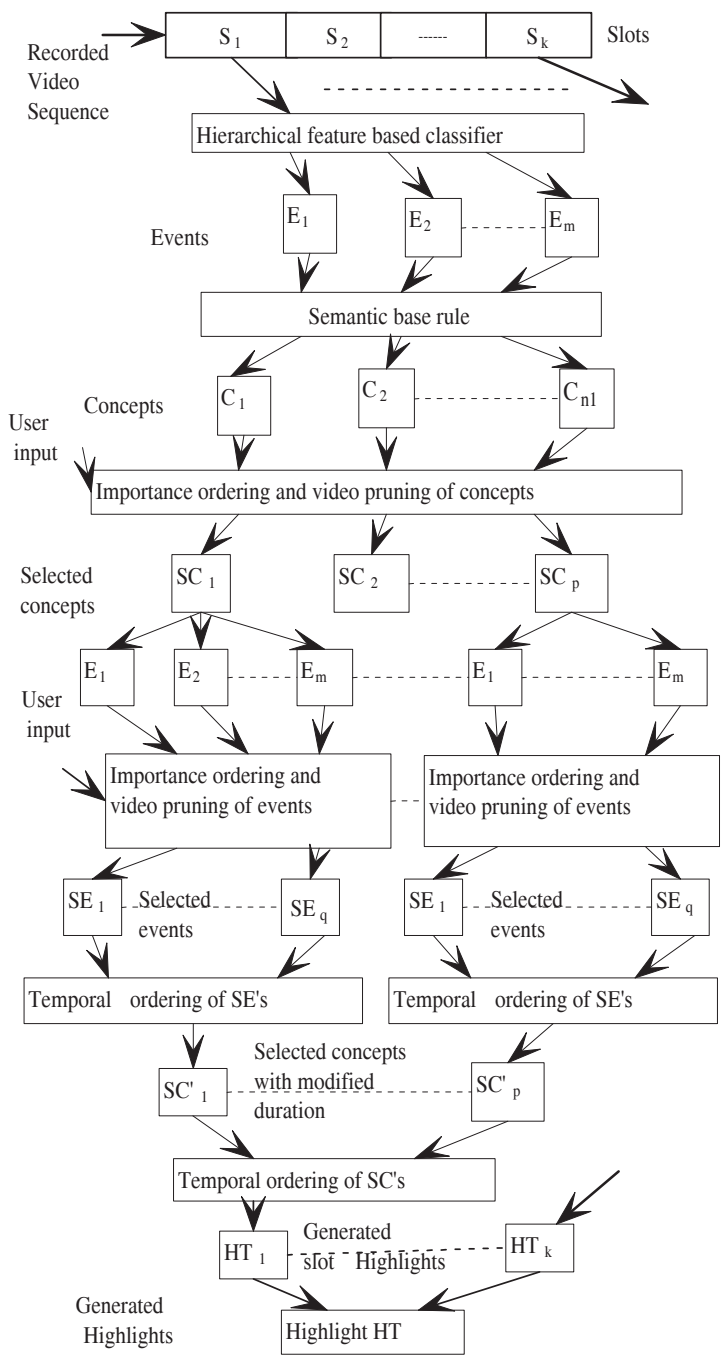

Fig. 1. Role of events and concepts in automated highlight generation

which must necessarily be temporally ordered. It is therefore possible to express the concepts as $C_{i}=\cup_{j=1}^{m} E_{j}$, for $i=1,2, \ldots \ldots \ldots, n$ where, $n$ is the number of concepts and $m$ is the number of events associated with the $i^{t h}$ concept. $C=\left\{C_{i}\right\}_{i=1,2, \ldots, n}$ represent the set of all extracted concepts.

Figure 1 presents an overall summary of our approach towards automated highlight generation from the events and concepts. The full game video is partitioned into $k$ slots. The set of the extracted concepts $C_{s_{l}} \subset C$ for each slot $S_{l}$ are ordered in decreasing value of importance parameters. The video pruning block accepts editor's choice, like editor's ranking of concept classes, total duration of highlights required, degree of abstraction required etc and outputs a set of pruned concepts $S C_{s_{l}}=\left\{S C_{s_{l} b}\right\}_{b=1,2, \ldots p}$, where $S C_{s_{l}} \subset C_{s_{l}}$ and $p<n_{l}$. The duration of these elements of
$S C_{s_{l}}$ is reduced by removing non-important events to generate the set $S C_{s_{l}}^{\prime}=\left\{S C_{s_{l} b}^{\prime}\right\}_{b=1,2, \ldots p}$. The elements of $S C_{s_{l}}^{\prime}$ are temporarily ordered and concatenated to generate the slothighlights $H T_{s_{l}}$. All these slot-highlights are concatenated in their temporal order to generate the highlight HT. Other than one-time input of editor's choice before highlight preparation, our proposed system doesn't require any manual intervention for highlight generation.

\section{HIGHLIGHT GENERATION ALGORITHM}

We define $C=\left\{C_{i}\right\}_{i=1}^{n}$, where, $n$ is the total number of concepts extracted from the full game video.

Let concept $C_{i}$ be of duration $d_{c_{i}}$

$H$ : required duration of the highlight

$\eta$ : degree of abstraction, $(0<\eta<1)$

$C_{T}$ : total duration of the extracted concepts, $\left(=\sum_{i=1}^{n} d_{c_{i}}\right)$

$C_{h}$ : total duration of the concepts selected for inclusion in highlights, $(H / \eta),\left(C_{h}<C_{T}\right)$

Step-1: Partition the cricket game into $k$ slots $S_{1}, S_{2}, \ldots \ldots, S_{k}$. Let $H_{1}, H_{2}, \ldots . ., H_{k}$ be the highlight durations of the slots $S_{1}, S_{2}, \ldots ., S_{k}$ respectively.

$$
H_{1}+H_{2}+\ldots .+H_{k}=H
$$

Let $n_{1}, n_{2}, \ldots \ldots, n_{k}$ be the number of concepts belonging to the slots $S_{1}, S_{2}, \ldots ., S_{k}$ respectively.

$$
n_{1}+n_{2}+\ldots .+n_{k}=n
$$

Let $C_{s_{l}}$ be the set of concepts belonging to slot $S_{l}$.

$$
C_{s_{l}} \subset C \quad \text { for }(1 \leq l \leq k)
$$

The duration of all the concepts belonging to slot $S_{l}$ is given by

$$
d_{s_{l}}=\sum_{i=x+1}^{x+n_{l}} d_{c_{i}}, \text { where } x=\sum_{f=1}^{l-1} n_{f}
$$

and

$$
C_{T}=\sum_{l=1}^{k} d_{s_{l}}
$$

Let $H_{l}$ be the allocated duration for the slot $S_{l}$.

$$
H_{l}=\frac{d_{s_{l}}}{C_{T}} * C_{h}
$$

Step-2: Select the concepts $S C_{s_{l}} \subset C_{s_{l}}$ according to their degree of importance such that the total duration of these selected concepts should be close to $C_{h_{l}}\left(=H_{l} / \eta\right)$.

Step-3: Let $S C_{s_{l b}}=\left\{E_{1}, E_{2}, E_{3}, \ldots \ldots E_{q}\right\}, \quad$ for $b=$ $1,2, \ldots, p$

where $S C_{s_{l b}} \in S C_{s_{l}}$ and $q=$ total number of events comprising the concept $S C_{s_{l b}}$. 
Select the set of events $S E_{j} \subset S C_{s_{l b}}, \quad(1 \leq j \leq q)$ of concept $S C_{s_{l b}}$ according to their degree of importance such that the total duration of the selected events is $d_{s c_{s_{l b}}}^{\prime}$.

$$
d_{s c_{s_{l b}}}^{\prime}=\eta * d_{s c_{s_{l b}}}
$$

where, $d_{s c_{s_{l b}}}$ is the duration of the selected concept, and $d_{s c_{s l b}}^{\prime}$ is the modified duration of the selected concept.

Step-4: Arrange all selected events $S E_{j}$ of concept $S C_{s_{l b}}$ in their temporal order to generate modified concept $S C_{s_{l b}}^{\prime}$.

$$
S C_{s_{l b}}^{\prime}=\bigcup_{S E_{j} \subset S C_{s_{l b}}} S E_{j}
$$

Step-5: Arrange all the modified concepts $S C_{s_{l b}}^{\prime}$ in their temporal order to generate the highlight $H T_{s_{l}}$ for slot $S_{l}$.

$$
H T_{s_{l}}=\bigcup_{b=1}^{p} S C_{s_{l b}}^{\prime}
$$

Step-6: Arrange the slot highlights $H T_{s_{l}}$ in their temporal order to generate the highlight $H T$.

$$
H T=\bigcup_{l=1}^{k} H T_{s_{l}}
$$

\section{SELECTION OF CONCEPTS AND EVENTS}

\subsection{Importance degree of the concepts}

The philosophy of the highlight is to show the concepts in the game according to their importance degree. We have estimated the importance of the concept in terms of concept rank and the duration of the concept.

Concept Rank: The rank based on importance degree of an concept $C_{i},\left(0 \leq I_{r}\left(C_{i}\right) \leq 1\right)$ is computed as follows:

$$
I_{r}\left(C_{i}\right)=\frac{1}{\sqrt{1+\left(\frac{2 r_{i}}{C}\right)^{2}}}
$$

where, $r_{i},\left(1 \leq r_{i} \leq C\right)$ denotes the rank of the $i^{t h}$ concept $C_{i}, C$ denotes the total number of the classes of the concepts in the video.

The importance degree defined as above, assigns almost equal importance to the top rankers in the concept classes and gradually decreases the importance for lower rankers (higher values of $r_{i}$ ).

Concept Duration: It is observed that the long duration concepts are important one. We define duration based importance degree of the concepts $C_{i},\left(0 \leq I_{d}\left(C_{i}\right) \leq 1\right)$ as follows:

\begin{tabular}{|c|c|c|}
\hline $\begin{array}{c}\text { Slot } \\
S_{l}\end{array}$ & $\begin{array}{l}\text { Concept } C_{i} \text { (Class: } \\
\text { Description) } / I\left(C_{i}\right)\end{array}$ & $\begin{array}{l}\text { Duration }(\mathrm{sec}) / \\
\text { start time - end time }\end{array}$ \\
\hline \multirow{8}{*}{$S_{1}$} & $C_{1}$ (hit:boundary)/0.20 & $31 / 1: 08-1: 39$ \\
\hline & $C_{2}$ (wicket:appeal)/0.52 & $61 / 4: 15-5: 16$ \\
\hline & $C_{3}$ (wicket:fine miss)/0.29 & $34 / 5: 52-6: 26$ \\
\hline & $C_{4}$ (hit:boundary)/0.21 & $32 / 6: 28-7: 00$ \\
\hline & $C_{5}$ (hit:leg-bye) $/ 0.236$ & $36 / 11: 52-12: 28$ \\
\hline & $C_{6}$ (hit:1-run)/0.242 & $37 / 15: 16-15: 53$ \\
\hline & $C_{7}$ (wicket:catch-out)/0.31 & 37/18:28-19:09 \\
\hline & $C_{8}$ (hit:3-runs)/0.242 & $37 / 22: 28-23: 05$ \\
\hline \multirow{5}{*}{$S_{2}$} & $C_{9}$ (hit:boundary)/0.187 & $28 / 25: 17-25: 45$ \\
\hline & $C_{10}$ (hit:3-runs)/0.336 & 51/33:17-34:08 \\
\hline & $C_{11}$ (hit:boundary)/0.105 & $16 / 37: 19-37: 35$ \\
\hline & $C_{12}$ (wicket:lbw-appeal)/0.26 & 31/39:02-39:33 \\
\hline & $C_{13}$ (hit:3-runs)/0.281 & 43/40:05-40:48 \\
\hline \multirow[t]{2}{*}{$S_{3}$} & $C_{14}$ (wicket:catch-out)/0.361 & 43/1:00:52-1:01:35 \\
\hline & $C_{15}$ (wicket:lbw)/0.566 & 67/1:04:05-1:05:12 \\
\hline \multirow{4}{*}{$S_{4}$} & $C_{16}$ (hit:boundary)/0.11 & 17/1:07:37-1:07:54 \\
\hline & $C_{17}$ (hit:boundary)/0.149 & 23/1:14:55-1:16:08 \\
\hline & $C_{18}$ (wicket:catch-out) $/ 0.38$ & 44/1:15:24-1:16:08 \\
\hline & $C_{19}$ (hit:boundary)/0.094 & 14/1:28:48-1:29:02 \\
\hline
\end{tabular}

$$
I_{d}\left(C_{i}\right)=\left(\frac{d_{c_{i}}}{d_{c_{i}}, \max }\right)_{c_{i} \in c_{s_{i}}}
$$

Importance degree of the concept: The importance degree of the concept is computed as: $I\left(C_{i}\right)=I_{r}\left(C_{i}\right) * I_{d}\left(C_{i}\right)$
Table 1. Details of the extracted concepts

\subsection{Importance degree of the events}

The concept is composed of the events. These events are selected according to their importance to achieve more concise highlights. The importance of the events is computed using the event rank and event occurrence time.

Event Rank: The rank based importance degree of an event $E_{j},\left(0 \leq I_{r}\left(E_{j}\right) \leq 1\right)$ is computed as follows:

$$
I_{r}\left(E_{j}\right)=\frac{1}{\sqrt{1+\left(\frac{2 r_{j}}{E}\right)^{2}}}
$$

where, $r_{j},\left(1 \leq r_{j} \leq E\right)$ denotes the rank of the $j^{\text {th }}$ events $E_{j}, E$ denotes the total number of the classes of the events in the video.

Event Occurrence Time: The events occurring at the final stage of the concepts such as replay, graphics animation of LBW, etc are of great importance. We define occurrence time based importance degree of the event $E_{j},\left(0 \leq I_{t}\left(E_{j}\right) \leq 1\right)$ as follows:

$$
I_{t}\left(E_{j}\right)=\frac{1}{\sqrt{1+\left(\frac{1}{j}\right)}} \quad \text { for }(1 \leq j \leq m)
$$

where, $m$ is the total number of extracted events.

Importance degree of an event: The importance degree of an event is computed as: $I\left(E_{j}\right)=I_{r}\left(E_{j}\right) * I_{t}\left(E_{j}\right)$ 
Table 2. Concept selected for highlight duration of $300 \mathrm{sec}$ with $\eta=0.75$

\begin{tabular}{|c|c|c|c|}
\hline$S_{l}$ & $\begin{array}{c}\text { Selected Concept: } \\
\text { duration }\left[C_{h_{l}}\right]\end{array}$ & $\begin{array}{c}\text { Selected Concept: } \\
\text { modified duration }\end{array}$ & $\begin{array}{l}H_{l} \\
(\mathrm{sec})\end{array}$ \\
\hline$S_{1}$ & $C_{2}: 61, C_{7}: 37$, & $C_{2}: 45.75, C_{3}: 25.5$, & \\
& $C_{3}: 34, C_{8}: 37$, & $C_{6}: 7.42, C_{7}: 27.75$, & \\
& $C_{6}: 9.89[178.89]$ & $C_{8}: 27.75$ & 134.17 \\
\hline$S_{2}$ & $C_{10}: 51, C_{13}: 43$, & $C_{10}: 38.25, C_{12}: 3.84$, & \\
& $C_{12}: 5.12[99.12]$ & $C_{13}: 32.25$ & 74.34 \\
\hline$S_{3}$ & $C_{15}: 64.52[64.52]$ & $C_{15}: 48.39$ & 48.39 \\
\hline$S_{4}$ & $C_{18}: 44$, & $C_{17}: 10.11, C_{18}: 33$ & \\
& $C_{17}: 13.48[57.48]$ & & 43.11 \\
\hline
\end{tabular}

Table 3. Importance degree of events for concept $C_{7}$

\begin{tabular}{|c|c|c|c|l|}
\hline$E_{j}$ & Class $/ r_{j} /$ Duration $(\mathrm{sec})$ & $I_{r}\left(E_{j}\right)$ & $I_{t}\left(E_{j}\right)$ & $I\left(E_{j}\right)$ \\
\hline$E_{1}$ & real-time (wicket)/1/7.9 & 0.96 & 0.71 & 0.68 \\
$E_{2}$ & bowler/2/1.8 & 0.87 & 0.82 & 0.71 \\
$E_{3}$ & batsman/3/0.6 & 0.76 & 0.87 & 0.66 \\
$E_{4}$ & Fielders' celebration/6/3.3 & 0.5 & 0.89 & 0.45 \\
$E_{5}$ & spectator/7/1.4 & 0.45 & 0.91 & 0.41 \\
$E_{6}$ & Fielders' celebration/6/5.9 & 0.5 & 0.93 & 0.46 \\
$E_{7}$ & batsman/3/5.7 & 0.76 & 0.94 & 0.71 \\
$E_{8}$ & replay/4/10.4 & 0.66 & 0.94 & 0.62 \\
\hline
\end{tabular}

\section{IMPLEMENTATION AND RESULTS}

We have experimented with 54 hours of live recordings of cricket video. Considering the space limitation of the paper, we are demonstrating our approach with 1 hour 30 minutes cricket recording of first 21 overs of the TV broadcast of India versus Australia International one-day cricket match . From this video, our hierarchical feature-based classifier have extracted 19 concepts [6], [7]. The total duration of all the extracted concepts is $11 \mathrm{~min} 22 \mathrm{sec}$. The concepts are ranked as follows: special moments (century, half century, achieving records, controversy, etc)-1, wicket-2, hit-3, presentation ceremony-4. We have partitioned this video into 4 slots where first 3 slots contain 5 overs and last slot contains 6 overs as shown in Table 1. The extracted concepts within each slots are arranged in their descending order of the degree of importance. Table 2 presents the selected concepts for highlight $H T$ of $5 \mathrm{~min}$ (i.e. $300 \mathrm{sec}$ ) from this video with $\eta=0.75$.

The events of concept are ranked as follows: real-time (hit/wicket)-1, bowler-2, batsman-3, replay-4, umpire-5, fielders' celebration-6, spectator- 8 . Table- 3 presents the degree of importance of the events for concept $C_{7}$ of the abovementioned cricket video. The events are arranged in the descending order of their degree of importances. Figure 2 shows the selected events $E_{1}, E_{2}, E_{3}, E_{6}, E_{7}, E_{8}$ for concept $C_{7}$ and their durations in seconds are 7.9, 1.8, 0.6, 1.35, 5.7, 10.4 respectively.

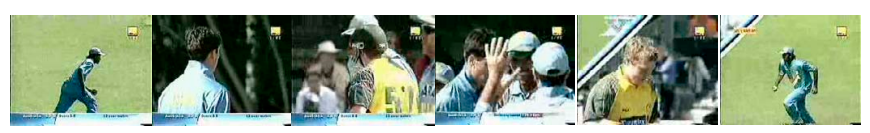

Fig. 2. The end frames of the selected events for concept $C_{7}$

\section{CONCLUSION AND FUTURE WORK}

In this paper, we have presented the game specific concept selection and event selection criteria. We have proposed the degree of abstraction parameter which decides how concisely the concepts can be produced in the highlights. This has created a new scope to generate a customized and automatic cricket video highlights. This approach can not only be extended to other sports, but also to other type of videos such as news, movies, etc. for video summarization applications.

\section{REFERENCES}

[1] B. Li and M. I. Sezan, "Semantic sports video analysis: approaches and new applications," in Proc of IEEE Int. Conf. on Image Processing, vol. 1, pp. 17-20, 2003.

[2] Y. Takahashi, N. Nitta, and N. Babaguchi, "Video summarization for large sports video archives," in Proc of IEEE Int. Conf. on Multimedia and Expo (ICME), pp. 1170 - 1173, July 2005.

[3] A. Hanjalic, "Adaptive extraction of highlights from a sport video based on excitement modeling," in IEEE Transactions on Multimedia, vol. 7, no. 6, pp. 1114 1122, 2005.

[4] O. Utsumi, K. Miura, I. Ide, S. Sakai, and H. Tanaka, "An object detection method for describing soccer games from video," in Proc of ICME, vol. 1, pp. 45-48, 2002.

[5] W. Hua, M. Han, and Y. Gong, "Baseball scene classification using multimedia features," in Proc of ICME, vol. 1, pp. 821-824, 2002.

[6] M.H.Kolekar and S. Sengupta, "A hierarchical framework for generic sports video classification," in Lecture Notes on Computer Science (LNCS), Springer-Verlag Berlin Heidelberg, vol. 3852, pp. 633-642, Jan 2006.

[7] M.H.Kolekar and S. Sengupta, "Hierarchical structure for audio-video based semantic classification of sports video sequences," in Proc of SPIE Int. Conf. on Visual Communications and Image Processing, Beijing, China, vol. SPIE 5960, pp. 401-409, July 2005. 